\title{
Modeling and Analysis of Epidemic Diffusion with Population Migration
}

\author{
Ming Liu and Yihong Xiao \\ Department of Management Science and Engineering, Nanjing University of Science and Technology, Nanjing 210094, China \\ Correspondence should be addressed to Ming Liu; liumingseu@126.com
}

Received 15 June 2013; Revised 24 July 2013; Accepted 30 July 2013

Academic Editor: Zhiwei Gao

Copyright ( 2013 M. Liu and Y. Xiao. This is an open access article distributed under the Creative Commons Attribution License, which permits unrestricted use, distribution, and reproduction in any medium, provided the original work is properly cited.

\begin{abstract}
An improved Susceptible-Infected-Susceptible (SIS) epidemic diffusion model with population migration between two cities is modeled. Global stability conditions for both the disease-free equilibrium and the endemic equilibrium are analyzed and proved. The main contribution of this paper is reflected in epidemic modeling and analysis which considers unequal migration rates, and only susceptible individuals can migrate between the two cities. Numerical simulation shows when the epidemic diffusion system is stable, number of infected individuals in one city can reach zero, while the number of infected individuals in the other city is still positive. On the other hand, decreasing population migration in only one city seems not as effective as improving the recovery rate for controlling the epidemic diffusion.
\end{abstract}

\section{Introduction}

As mentioned in Rachaniotis et al. [1], a serious epidemic is a problem that tests the ability of a nation to effectively protect its population, to reduce human loss, and to rapidly recover. Sometime such a problem may acquire worldwide dimensions. For example, during the period from November 2002 to August 2003, 8422 people in 29 countries were infected with SARS; 916 of them were dead at last for the effective medical resources appeared late. Other diseases, such as HIV and H1N1, can also cause significant numbers of direct infectious disease deaths. Epidemic diffusion is a typical complex dynamic system problem in Gao et al. [2], for we do not know what kind of epidemic outbreaks, when it outbreaks, and how it diffuses. Generally, after an epidemic outbreaks, public officials are faced with many critical and complex issues, the most important of which is to make certain how the epidemic diffuses so that the rescue operation efficiency is maximized.

Traditionally, analytical works on epidemic diffusion are concentrated on the compartmental epidemic models of ordinary differential equations (Mishra and Saini [3]; Sun and Hsieh [4]; Li et al. [5]; Zhang et al. [6]; Zhang and Ma [7]). In these models, the total population is divided into several independence classes, and each class of individuals is closed into a compartment. The sizes of the compartments are large enough, and the mixing of members is homogeneous. In other words, the models based on the differential equations are always under the assumption of both homogeneous infectivity and homogeneous connectivity of each individual. Recently, Kim et al. [8] described the transmission of avian influenza among birds and humans. The behavior of positive solutions to a reaction diffusion system with homogeneous Neumann boundary conditions was investigated in their work. Liu and Zhang [9] presented an SEIRS epidemic model on the scale-free networks, where the active contact number of each vertex was assumed to be either constant or proportional to its degree in their model. Samsuzzoha et al. [10] used a diffusive epidemic model to describe the transmission of influenza. The equations were solved numerically by using the splitting method under different initial distribution of population density. Further, Samsuzzoha et al. [11] presented a vaccinated diffusive compartmental epidemic model to explore the impact of vaccination as well as diffusion on the transmission dynamics of influenza. A very recent research by Shi and Dong [12] formulates and discusses models for the spread of infectious diseases with variable population sizes and vaccinations on the susceptible individuals.

The other stream of related research to our work is on the epidemic diffusion with population migration. For instance, 
Hethcote [13] proposed that deterministic communicable disease models were initial value problems for a system of ordinary differential equations, and thus he considered the asymptotic stability for the equilibrium points for models involving temporary immunity, disease-related fatalities, carriers, migration, dissimilar interacting groups, and transmission by vectors. In his work, both susceptible individuals and infected individuals in each population could migrate (only equal rates were considered), which led to different equilibriums. Another model that considers two interacting populations undergoing SIS dynamics was presented in Kribs-Zaleta and Velasco-Hernández [14]. The authors considered that the two groups may have different values for model parameters especially those dealing with vaccination. Liebovitch and Schwartz [15] proposed that classical disease models always use a mass action term as the interaction between infected and susceptible people in separate patches, and they derived the equations when this interaction is a migration of people between patches. Sani and Kroese [16] formulated various mathematical control problems for HIV spread in mobile heterosexual populations. They applied the cross-entropy method to solve these highly multimodal and nonlinear optimization problems, and demonstrated the effectiveness of the method via a range of experiments and illustrated how the form of the optimal control function depends on the mathematical model used for the HIV spread. Yang et al. [17] considered SIR and SIS epidemic models with bilinear incidence and migration between two patches, where infected individuals cannot migrate from one patch to another due to medical screening. They found the thresholds classifying the global dynamics of the models in terms of the model parameters, and they obtained the global asymptotical stability of the disease free and the disease-endemic equilibrium. Wolkewitz and Schumacher [18] pointed out that the main limitation of the compartmental models is that several parameters are based on uncertain expert guesses (default values) and are not estimated from the study data. Lee et al. [19] extended the SEIR model to incorporate population migration between cities and investigated the effectiveness of travel restrictions as a control against the spread of influenza.

It is worth mentioning that majority of the existing studies relies on different kinds of differential equations. For instance, first-order partial differential equations are used to integrate the age structures; second-order partial differential equations are suitable when a diffusion term exists; integral differential equations or differential equations are often used when time delay or delay factors are considered. These are the typical methods to model and analyze the complex dynamic system of epidemic diffusion. As a continued work of our previous paper in [20], this paper presents an SIS epidemic model with population migration between two cities. We consider unequal migration rates for these two populations, and only susceptible individuals can migrate, which is different from the whole existing works. The remainder of the paper is organized as follows. Section 2 proposes the SIS epidemic diffusion model, which considers susceptible individuals migration between two cities. In Section 3, the dynamic characteristics of the proposed SIS model are analyzed. Numerical simulation and key parameters analysis are presented in

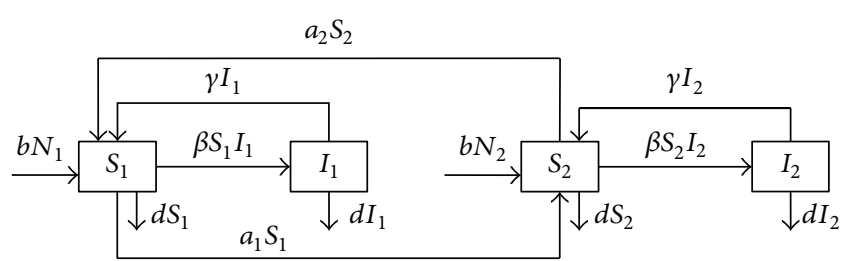

FIGURE 1: The transfer diagram of SIS model with population migration.

Section 4. Finally, Section 5 discusses the conclusions and suggests for the future research.

\section{Epidemic Diffusion Model (SIS)}

Since the compartment model of epidemic diffusion is a mature theory, herein we omit the verbose introduction of the framework process. In this paper, we divide people in epidemic areas into two groups: susceptible individuals $(S)$ and infected individuals $(I)$. The transfer diagram of individuals in the epidemic areas can be illustrated as Figure 1.

To smooth the formulation progress of the SIS epidemic diffusion model in the following subsections, some assumptions and parameters are specified as follows.

(1) The susceptible individuals and the infected individuals in city $i$ at time $t$ are denoted as $S_{i}(t)$ and $I_{i}(t)$, respectively. Thus, the total individuals in city $i$ are $N_{i}(t)=S_{i}(t)+I_{i}(t), \quad i=1,2$.

(2) $b$ and $d$ are the natural birth rate and the natural death rate, respectively. $\gamma$ is the recovery rate. $\beta$ is the propagation coefficient. To facilitate the process in the following sections, we assume that $b=d$. Moreover, disease-related death rate is not considered in this work.

(3) Only the susceptible individuals can migrate in this paper. $a_{i}$ represents the migrating-out rate of susceptible individuals in city $i\left(a_{i}>0\right.$ for $i=1,2$ and $\left.a_{1} \neq a_{2}\right)$.

(4) Using the notation $N$ to represent the total number of the population in these two cities, $N=N_{1}+N_{2}$. Note that $N$ is a constant.

Hence, the ordinary differential equations for the SIS epidemic diffusion model can be formulated as

$$
\begin{aligned}
& \frac{d S_{1}}{d t}=b N_{1}-a_{1} S_{1}+a_{2} S_{2}-d S_{1}-\beta S_{1} I_{1}+\gamma I_{1}, \\
& \frac{d I_{1}}{d t}=\beta S_{1} I_{1}-\gamma I_{1}-d I_{1}, \\
& \frac{d S_{2}}{d t}=b N_{2}+a_{1} S_{1}-a_{2} S_{2}-d S_{2}-\beta S_{2} I_{2}+\gamma I_{2}, \\
& \frac{d I_{2}}{d t}=\beta S_{2} I_{2}-\gamma I_{2}-d I_{2} .
\end{aligned}
$$

ODE (1) describes the following dynamics of epidemic diffusion among the population groups. (1) The change rate 
of the susceptible population in both city 1 and city 2 is determined by the entry population, the exiting population, and the losing population who actually gets exposed to the disease and thus is counted towards the class of infected population. The last one is in proportion to the propagation coefficient $\beta$ and both of the current mass of the susceptible individuals and the current mass of the infected individuals. (2) The change rate of the infected population is determined by the difference between the entering population, those of the susceptible population who get sick, the exiting population, and the losing population. All parameters $\beta, b, \gamma, a_{1}$, and $a_{2}$ are positive, and initial conditions for the model are demonstrated as follows:

$$
\begin{gathered}
I_{1}(0)=i_{1}^{0} \ll N, \quad I_{2}(0)=i_{2}^{0} \ll N, \\
S_{1}(0)=s_{1}^{0}, \quad S_{2}(0)=N-s_{1}^{0}-i_{1}^{0}-i_{2}^{0} .
\end{gathered}
$$

\section{Model Analysis}

3.1. Condition of the Epidemic Diffusion. As shown above, $I_{1}(0)=i_{1}^{0} \ll N, I_{2}(0)=i_{2}^{0} \ll N, S_{1}(0)=s_{1}^{0}$, and $S_{2}(0)=N-s_{1}^{0}-i_{1}^{0}-i_{2}^{0}$ are initial conditions for the proposed model, which symbolize the initial number of susceptible and infected individuals. Then, it is easy to obtain the initial condition for epidemic diffusion, which should satisfy the following premise:

$$
\left.\frac{d I_{1}}{d t}\right|_{t=0}>0 \quad \text { or }\left.\quad \frac{d I_{2}}{d t}\right|_{t=0}>0
$$

Taking it into (1), we can obtain the initial condition of the susceptible individuals in city 1 and city 2 :

$$
s_{1}^{0}>\frac{b+\gamma}{\beta} \text { or } s_{2}^{0}<N-i_{1}^{0}-i_{2}^{0}-\frac{b+\gamma}{\beta} .
$$

Equation (4) shows that the spread of epidemic happens only when $s_{1}^{0}$ and $s_{2}^{0}$ meet the previous initial conditions.

3.2. Existence of the System Equilibrium Solution. Generally, it is difficult to obtain the analytic solution of (1). To analyze the epidemic diffusion, we consider the stable state of (1). Considering that $b=d$ and expunging $S_{2}$, (1) can be rewritten as

$$
\begin{aligned}
& \frac{d S_{1}}{d t}=-a_{1} S_{1}+a_{2}\left(N-S_{1}-I_{1}-I_{2}\right)-\beta S_{1} I_{1}+(b+\gamma) I_{1}, \\
& \frac{d I_{1}}{d t}=\beta S_{1} I_{1}-(b+\gamma) I_{1}, \\
& \frac{d I_{2}}{d t}=\beta\left(N-S_{1}-I_{1}-I_{2}\right) I_{2}-(b+\gamma) I_{2} .
\end{aligned}
$$

Letting $d I_{1} / d t=0$, we can get $I_{1}=0$ or $S_{1}=(b+\gamma) / \beta$. Similarly, letting $d I_{2} / d t=0$, we can obtain $I_{2}=0$ or $S_{1}+I_{1}+$ $I_{2}=N-((b+\gamma) / \beta)$. With the partial derivatives $d S_{1} / d t=0$, $d I_{1} / d t=0$, and $d I_{2} / d t=0$, we can obtain one equilibrium point of the SIS epidemic diffusion system intuitively when $I_{1}=0$ and $I_{2}=0$ :

$$
P_{1}=\left(S_{1}, I_{1}, I_{2}\right)=\left(\frac{a_{2}}{a_{1}+a_{2}} N, 0,0\right) .
$$

From (6) we can see that both numbers of infected individuals in city 1 and city 2 are zero, which indicate that epidemic diffusion in these two cities does not happen, and all the individuals in these two cities are susceptible individuals at last. Herein, we call it disease-free equilibrium point.

When $I_{1}=0$ and $S_{1}+I_{1}+I_{2}=N-((b+\gamma) / \beta)$, we can obtain the second equilibrium point of the SIS epidemic diffusion system:

$$
P_{2}=\left(S_{1}, I_{1}, I_{2}\right)=\left(\frac{a_{2}}{a_{1}} \cdot \frac{b+\gamma}{\beta}, 0, N-\frac{a_{1}+a_{2}}{a_{1}} \cdot \frac{b+\gamma}{\beta}\right) .
$$

From (7), when the SIS epidemic diffusion system is stable, the number of infected individuals in city 1 is zero, and some infected individuals in city 2 still exist. In this condition, we call it the endemic equilibrium point.

Likewise, when $S_{1}=(b+\gamma) / \beta$ and $I_{2}=0$, we can obtain the third equilibrium point of the SIS epidemic diffusion system:

$$
P_{3}=\left(S_{1}, I_{1}, I_{2}\right)=\left(\frac{b+\gamma}{\beta}, N-\frac{a_{1}+a_{2}}{a_{2}} \cdot \frac{b+\gamma}{\beta}, 0\right) .
$$

In line with the above work, when the SIS epidemic diffusion system is stable, the number of infected individuals in city 2 is zero, and some infected individuals in city 1 still exist. So it is called endemic equilibrium point as well.

It is worth mentioning that, when $S_{1}=(b+\gamma) / \beta$ and $S_{1}+I_{1}+I_{2}=N-((b+\gamma) / \beta)$, there is $d S_{1} / d t=\left(a_{2}-a_{1}\right) \cdot(b+$ $\gamma) / \beta \neq 0$ for that $a_{1} \neq a_{2}$. That means, under the conditions of $S_{1}=(b+\gamma) / \beta$ and $S_{1}+I_{1}+I_{2}=N-((b+\gamma) / \beta)$, there is no solution for the simultaneous equations $d S_{1} / d t=0, d I_{1} / d t=$ 0 , and $d I_{2} / d t=0$.

\subsection{Stability of the System Equilibrium Solution}

Lemma 1. Disease-free equilibrium point $P_{1}$ in the SIS epidemic diffusion system is stable only when $\beta<\min \left\{\left(a_{1}+a_{2}\right)(b+\right.$ $\left.\gamma) / a_{1} N,\left(a_{1}+a_{2}\right)(b+\gamma) / a_{2} N\right\}$.

Proof. Letting $P=d S_{1} / d t, Q=d I_{1} / d t$, and $R=d I_{2} / d t$, the Jacobi matrix of (5) can be obtained as follows: 


$$
J=\left(\begin{array}{lll}
\frac{\partial P}{\partial S_{1}} & \frac{\partial P}{\partial I_{1}} & \frac{\partial P}{\partial I_{2}} \\
\frac{\partial Q}{\partial S_{1}} & \frac{\partial Q}{\partial I_{1}} & \frac{\partial Q}{\partial I_{2}} \\
\frac{\partial R}{\partial S_{1}} & \frac{\partial R}{\partial I_{1}} & \frac{\partial R}{\partial I_{2}}
\end{array}\right)=\left(\begin{array}{ccc}
-a_{1}-a_{2}-\beta I_{1} & b+\gamma-a_{2}-\beta S_{1} & -a_{2} \\
\beta I_{1} & \beta S_{1}-(b+\gamma) & 0 \\
-\beta I_{2} & -\beta I_{2} & \beta\left(N-S_{1}-I_{1}-2 I_{2}\right)-(b+\gamma)
\end{array}\right)
$$

For $P_{1}=\left(S_{1}, I_{1}, I_{2}\right)=\left(a_{2} /\left(a_{1}+a_{2}\right) N, 0,0\right)$, the Jacobi matrix $J$ can be rewritten as follows:

$$
J_{P_{1}}=\left(\begin{array}{ccc}
-a_{1}-a_{2} & b+\gamma-a_{2}-\frac{a_{2} N}{a_{1}+a_{2}} \beta & -a_{2} \\
0 & \frac{a_{2} N}{a_{1}+a_{2}} \beta-(b+\gamma) & 0 \\
0 & 0 & \frac{a_{1} N}{a_{1}+a_{2}} \beta-(b+\gamma)
\end{array}\right)
$$

According to the Jacobi matrix $J_{P_{1}}$, it is easy to obtain the secular equation of (5):

$$
\begin{aligned}
(\lambda+ & \left.a_{1}+a_{2}\right)\left(\lambda+b+\gamma-\frac{a_{2} N}{a_{1}+a_{2}} \beta\right) \\
& \times\left(\lambda+b+\gamma-\frac{a_{1} N}{a_{1}+a_{2}} \beta\right)=0 .
\end{aligned}
$$

The three latent roots of this secular equation are $-a_{1}-$ $a_{2}, a_{2} N /\left(a_{1}+a_{2}\right) \beta-b-\gamma$, and $a_{1} N /\left(a_{1}+a_{2}\right) \beta-b-$ $\gamma$. Based on Routh-Hurwitz stability criterion, only when $\beta<\left(a_{1}+a_{2}\right)(b+\gamma) / a_{1} N$ and $\beta<\left(a_{1}+a_{2}\right)(b+\gamma) / a_{2} N$, three latent roots of the secular equation would have negative real parts, simultaneously, and then $P_{1}=\left(S_{1}, I_{1}, I_{2}\right)=$ $\left(a_{2} /\left(a_{1}+a_{2}\right) N, 0,0\right)$ is the stable solution of the differential equations.

Lemma 2. Endemic equilibrium point $P_{2}$ in the SIS epidemic diffusion system is stable only when $a_{2}<a_{1}$ and $\beta>\left(a_{1}+\right.$ $\left.a_{2}\right)(b+\gamma) / a_{1} N$.

Proof. As far as we are concerned, if the endemic equilibrium point $P_{2}=\left(S_{1}, I_{1}, I_{2}\right)=\left(a_{2} / a_{1} \cdot(b+\gamma) / \beta, 0, N-\left(a_{1}+a_{2}\right) / a_{1} \cdot(b+\right.$ $\gamma) / \beta$ ) exists, it should satisfy condition $I_{2}>0$ firstly. Namely, the propagation coefficient $\beta$ should satisfy $\beta>\left(a_{1}+a_{2}\right)(b+$ $\gamma) / a_{1} N$. Then, similar to Lemma 1 , we can obtain the Jacobi matrix for $P_{2}$ as follows:

$$
J_{P_{2}}=\left(\begin{array}{ccc}
-a_{1}-a_{2} & b+\gamma-a_{2}-\frac{a_{2}}{a_{1}}(b+\gamma) & -a_{2} \\
0 & \frac{a_{2}}{a_{1}}(b+\gamma)-(b+\gamma) & 0 \\
\frac{a_{1}+a_{2}}{a_{1}}(b+\gamma)-\beta N & \frac{a_{1}+a_{2}}{a_{1}}(b+\gamma)-\beta N & \frac{a_{1}+a_{2}}{a_{1}}(b+\gamma)-\beta N
\end{array}\right) .
$$

According to the Jacobi matrix $J_{P_{2}}$, we can obtain the secular equation of (5) again:

$$
\left[\lambda+b+\gamma-\frac{a_{2}}{a_{1}}(b+\gamma)\right]\left(\lambda^{2}+A_{1} \lambda+A_{0}\right)=0
$$

where $A_{0}=a_{1}\left[\beta N-\left(\left(a_{1}+a_{2}\right) / a_{1}\right)(b+\gamma)\right]$ and $A_{1}=a_{1}+$ $a_{2}+\beta N-\left(\left(a_{1}+a_{2}\right) / a_{1}\right)(b+\gamma)$. Obviously, one of the latent roots of (13) is $\lambda_{1}^{*}=\left(\left(a_{2}-a_{1}\right) / a_{1}\right)(b+\gamma)$. Only when $a_{2}<a_{1}$, the latent root $\lambda_{1}^{*}$ is negative. On the other hand, when $\beta>$ $\left(a_{1}+a_{2}\right)(b+\gamma) / a_{1} N$, there is $A_{0}>0$ and $A_{1}>0$. Based on Routh-Hurwitz stability criterion, the other two latent roots of (13) will be with negative real parts. Therefore, $P_{2}$ is the stable solution of the simultaneous differential equations only when $a_{2}<a_{1}$ and $\beta>\left(a_{1}+a_{2}\right)(b+\gamma) / a_{1} N$.

Lemma 3. Endemic equilibrium point $P_{3}$ in the SIS epidemic diffusion system is stable only when $a_{1}<a_{2}$ and $\beta>\left(\left(a_{1}+\right.\right.$ $\left.\left.a_{2}\right)(b+\gamma)\right) / a_{2} N$. 
Proof. Similar to Lemma 2, if the endemic equilibrium point $P_{3}=\left(S_{1}, I_{1}, I_{2}\right)=\left((b+\gamma) / \beta, N-\left(a_{1}+a_{2}\right) / a_{2} \cdot(b+\gamma) / \beta, 0\right)$ exists, it should satisfy condition $I_{1}>0$. That is, the propagation coefficient $\beta$ should satisfy $\beta>\left(a_{1}+a_{2}\right)(b+\gamma) / a_{2} N$. Then, we can obtain the Jacobi matrix for $P_{3}$ as follows:

$$
J_{P_{3}}=\left(\begin{array}{ccc}
-a_{1}-a_{2}-\beta N+\frac{a_{1}+a_{2}}{a_{2}}(b+\gamma) & -a_{2} & -a_{2} \\
\beta N-\frac{a_{1}+a_{2}}{a_{2}}(b+\gamma) & 0 & 0 \\
0 & 0 & \frac{a_{1}-a_{2}}{a_{2}}(b+\gamma)
\end{array}\right) \text {. }
$$

Again, according to the Jacobi matrix $J_{P_{3}}$, we can obtain the secular equation of (5):

$$
\left[\lambda-\frac{a_{1}-a_{2}}{a_{2}}(b+\gamma)\right]\left(\lambda^{2}+B_{1} \lambda+B_{0}\right)=0,
$$

where $B_{0}=a_{2}\left[\beta N-\left(\left(a_{1}+a_{2}\right) / a_{2}\right)(b+\gamma)\right]$ and $B_{1}=a_{1}+$ $a_{2}+\beta N-\left(\left(a_{1}+a_{2}\right) / a_{2}\right)(b+\gamma)$. Obviously, one of the latent roots of $(15)$ is $\lambda_{1}^{*}=\left(\left(a_{1}-a_{2}\right) / a_{2}\right)(b+\gamma)$. Only when $a_{1}<a_{2}$, the latent root $\lambda_{1}^{*}$ is negative. On the other hand, when $\beta>$ $\left(a_{1}+a_{2}\right)(b+\gamma) / a_{2} N$, there is $B_{0}>0$ and $B_{1}>0$. Based on Routh-Hurwitz stability criterion, the other two latent roots of (15) will be with negative real parts. Therefore, $P_{3}$ is the stable solution of the simultaneous differential equations only when $a_{1}<a_{2}$ and $\beta>\left(a_{1}+a_{2}\right)(b+\gamma) / a_{2} N$.

Remark 4. From Lemmas 1, 2, and 3, we can draw a conclusion that the diffusion threshold of the SIS epidemic diffusion model relies on the migrating-out coefficients of susceptible individuals of the two cities $a_{i}(i=1,2)$; it also depends on the three key parameters: the total individuals of the two cities $N$, the birth rate $b$, and the recovery rate $\gamma$.

\section{Numerical Simulation and Key Parameters Discussion}

In this section, we take a numerical simulation to test how well the proposed model may be applied in practice. The initial values of parameters in the proposed epidemic diffusion model are acquired from Liu and Zhao [21] and by interviews with public health care administrative personnel, which are given as follows: $\beta=8 \times 10^{-6}, b=2 \times 10^{-4}$, $\gamma=0.4, a_{1}=0.02, a_{2}=0.01, N=10^{5}, S_{1}(0)=0.7 \times$ $10^{5}, I_{1}(0)=600$, and $I_{2}(0)=400$. We use MATLAB 7.0 mathematical solver together with Runge-Kutta method to simulate the epidemic model. The test is performed on an Intel (R) Core (TM) i3 CPU $2.4 \mathrm{GHz}$ with $2 \mathrm{~GB}$ RAM under Microsoft Windows XP. Figure 2 is the evolution trajectories of the epidemic model. The curves, respectively, represent the different groups of people over time in these two cities.

From Figure 2, one can see that the evolution trajectories of the SIS epidemic model with population migration between two cities are complicated. The number of susceptible individuals in city 1 decreases gradually with time increasing, while the number of susceptible individuals in city 2 increases at first and then decreases. On the other hand, the number of infected individuals in city 1 increases at first and then decreases, while the number of infected individuals in city 2 increases gradually with time increasing. However, all the susceptible individuals and infected individuals in city 1 and city 2 tend to the fixed values when time is long enough $(t>300)$. Meanwhile, the limit value of the SIS epidemic diffusion model with population migration between two cities is $Q_{1}=$ $\left(S_{1}, S_{2}, I_{1}, I_{2}\right)=\left(2.5013 \times 10^{4}, 5.0025 \times 10^{4}, 0,2.4961 \times\right.$ $10^{4}$ ).

In line with the initial values we defined previously, we have $a_{2}<a_{1}$ and $\beta>\left(a_{1}+a_{2}\right)(b+\gamma) / a_{1} N$. According to Lemma 2 in Section 3, one can get that the number of susceptible and infected individuals will be converged at $Q_{2}=$ $\left(S_{1}^{*}, S_{2}^{*}, I_{1}^{*}, I_{2}^{*}\right)=\left(2.50125 \times 10^{4}, 5.00245 \times 10^{4}, 0,2.4963 \times\right.$ $\left.10^{4}\right)$. One can see that $Q_{1}$ is very close to $Q_{2}$, which is not a surprise, as it is consistent with the analytical conclusion in the last section. Once an epidemic outbreak occurs, we are more concerned with the change regularity of the infected individuals in practice. Therefore, in the following subsections, we will discuss the relationship between the key parameters and the number of the infected individuals.

Figure 3 demonstrates the change of the number of the infected individuals in both cities with different propagation coefficients. It is easy to know that the evolution trajectories of infected individuals in city 1 are different from those in city 2 for any certain propagation coefficient $\beta$. From Figure 3(a), we can see that, in the first some days, the larger $\beta$ is, the faster spread of the epidemic in city 1 is. However, from Figure 3(b), we cannot get the similar conclusion. Number of infected individuals in city 2 is not in direct proportion to the propagation coefficient $\beta$. It is worth mentioning that, when the number of infected individuals in city 1 reaches zero, the number of infected individuals in the other city is still positive when the epidemic diffusion system is stable. This is consistent with Lemma 2 in Section 3. Similarly, if the initial conditions changed, we can also test and verify the other lemmas.

Figure 4 demonstrates the change of the number of the infected individualss in both cities with different recovery rate $\gamma$. As shown in Figure 4(a), when $\gamma=0.2$, the maximum number of infected individuals in city 1 is about $3.3 \times 10^{4}$. 




FIGURE 2: Evolution trajectories of the SIS epidemic model.

When $\gamma=0.3$, the number is about $1.5 \times 10^{4}$. When $\gamma=0.4$, the maximum number of infected individual in city 1 is less than $0.5 \times 10^{4}$. It informs us that the larger the recovery rate constant is, the smaller of the maximum number of infected individuals in city 1 is. Similar phenomenon can also be observed from Figure 4(b) in city 2. Such figure informs us that it is important to improve the recovery rate as much as possible when controlling an epidemic spread.

Figure 5 shows the change of the number of infected individuals in both cities with different migrating-out coefficients $a_{1}$ and $a_{2}$. According to Figure 5, one can observe that no matter in city 1 or in city 2 , the evolution trajectories of the infected individuals may generate a serious change when the migrating-out coefficient of susceptible individuals changed. For example, in city 1 , when $a_{2}<a_{1}\left(a_{1}=0.02, a_{2}=0.01\right.$ and $\left.a_{1}=0.02, a_{2}=0.015\right)$, the number of infected individuals tends to be zero. However, when $a_{1}<a_{2}\left(a_{1}=0.02, a_{2}=\right.$ 0.025 and $\left.a_{1}=0.02, a_{2}=0.03\right)$, the number of infected individuals tends to be a positive constant above $1 \times 10^{4}$. In other words, with the increment of migrating-out coefficient in city 2 , the limit number of infected individuals in city 1 may become positive from zero. The larger the migratingout coefficient in city 2 is, the larger the limit number of infected individuals in city 1 is. Opposite to city 1 , when $a_{2}<a_{1}\left(a_{1}=0.02, a_{2}=0.01\right.$ and $\left.a_{1}=0.02, a_{2}=0.015\right)$, the number of infected individuals in city 2 tends to be a positive constant above $1 \times 10^{4}$. When $a_{1}<a_{2}\left(a_{1}=0.02\right.$, $a_{2}=0.025$ and $\left.a_{1}=0.02, a_{2}=0.03\right)$, the number of infected individuals is very small and tends to be zero at last. It informs us that with the increment of migrating-out coefficient in city 2, the limit number of infected individuals in city 2 may become zero from a positive value. The larger the migrating-out coefficient in city 2 is, the smaller the limit number of infected individuals in city 2 is. To summarize, decreasing the migration population in only one city is not as effective as improving the recovery rate for controlling the epidemic diffusion. However, we can find a trade-off between the migrating-out coefficients in these two cities and hence can control the infected individuals in both cities at last.

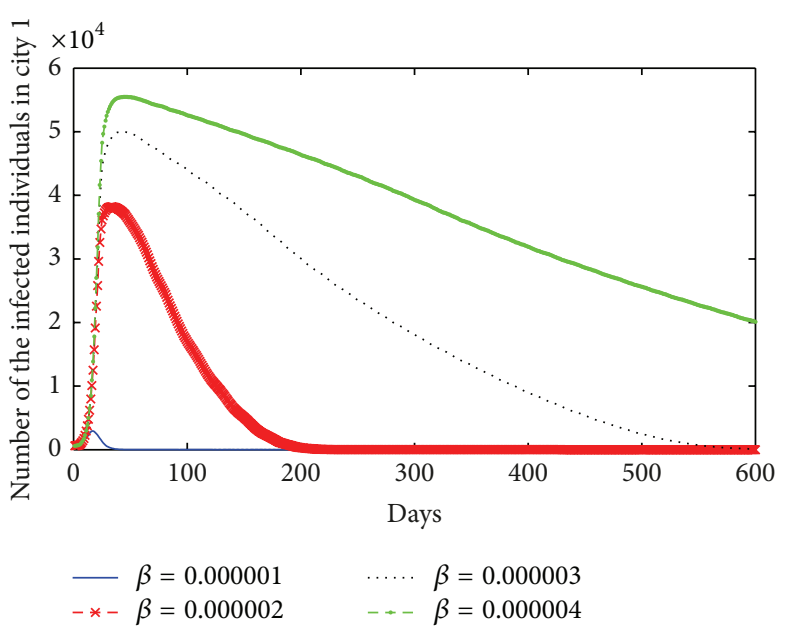

(a) City 1

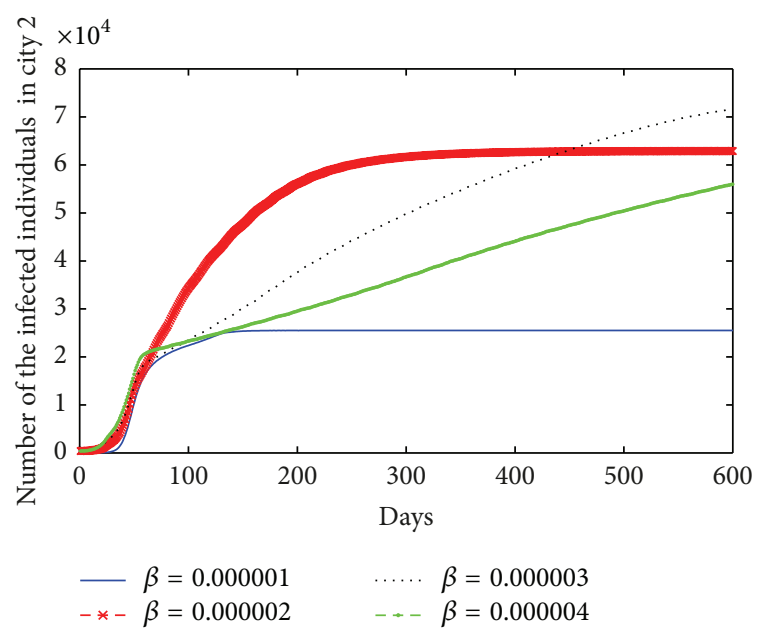

(b) City 2

Figure 3: Number of the infected individuals versus different $\beta$.

\section{Conclusions}

In this paper, we considered an SIS epidemic diffusion model with population migration between two cities, and proved that the model has one disease-free equilibrium point and two endemic equilibrium points. To summarize, our model differs from past researches in at least two aspects. Firstly, unequal migration rates between the two cities are considered; secondly, only the susceptible individuals can migrate between the two cities. Numerical simulation shows that number of infected individuals in one city can reach zero, while the number of infected individuals in the other city is still positive when the epidemic diffusion system is stable. One the other hand, decreasing population migration in only one city is not as effective as improving the recovery rate for controlling the epidemic diffusion.

As a limitation of the model, disease-related death rate of infected individuals in both cities was not considered. Actually, some epidemics would cause death, and thus consideration of a disease-related death rate would make it more accurate. On the other hand, epidemic diffusion model with population migration among more than two cities should also be taken into account. A discussion of possible extension to 


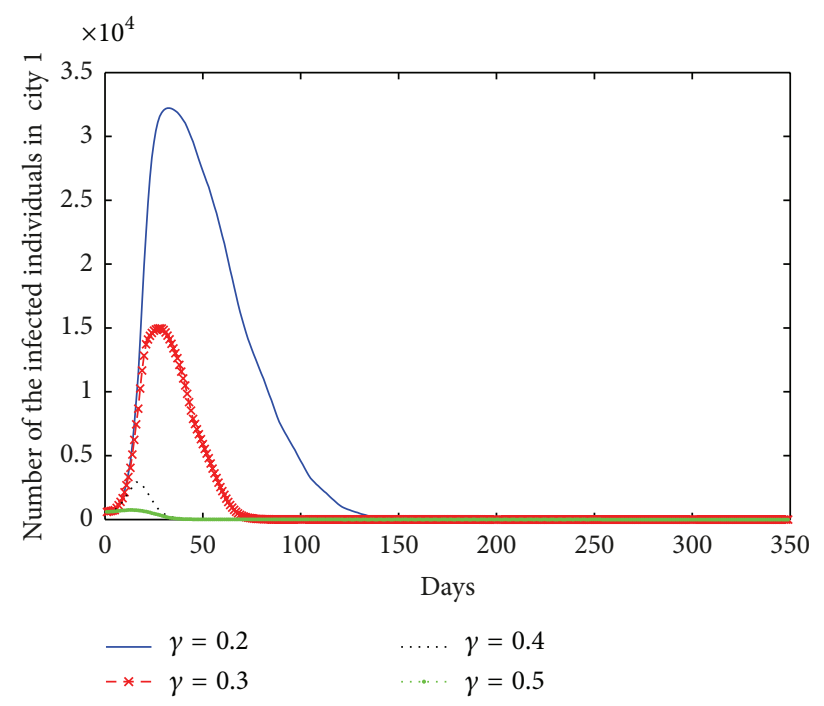

(a) City 1

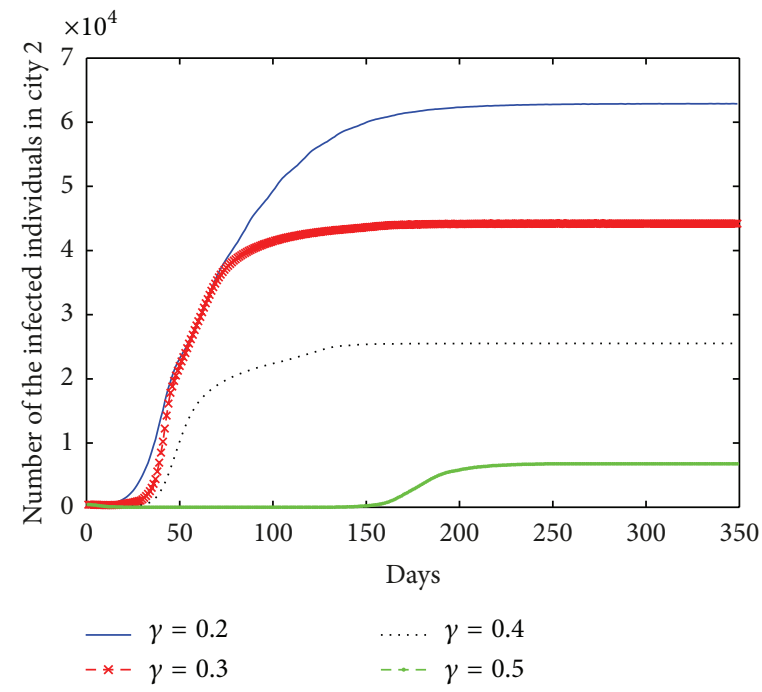

(b) City 2

FIGURE 4: Number of the infected individuals versus different $\gamma$.

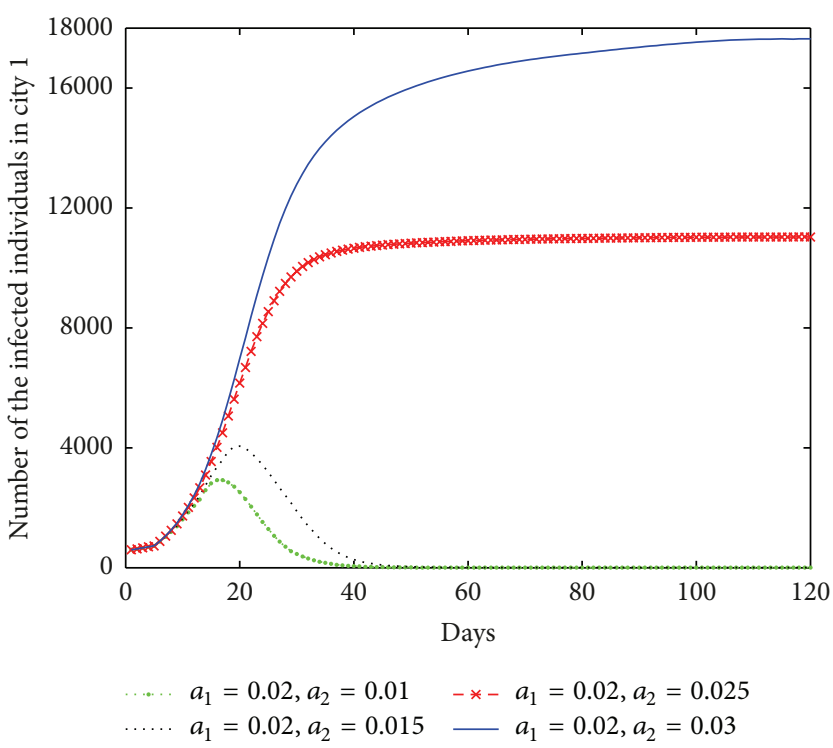

(a) City 1

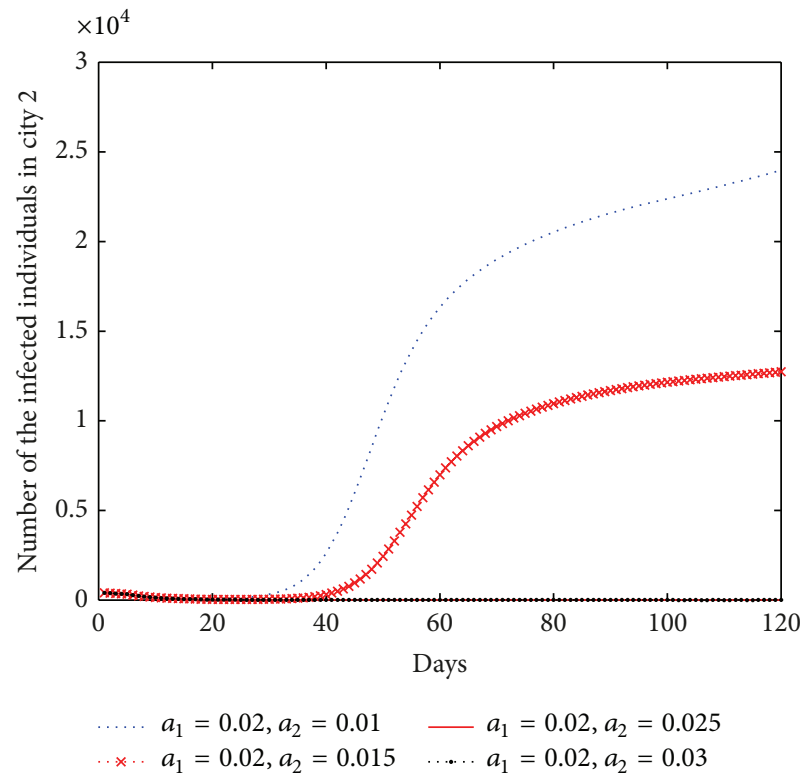

(b) City 2

FIGURE 5: Number of the infected individuals versus different $a_{1}$ and $a_{2}$.

more than two cities would be more realistic and the research would be more practical.

\section{Acknowledgments}

This work has been partially supported by the National Natural Science Foundation of China (no. 71301076), the MOE (Ministry of Education in China) Project of Humanities and Social Sciences (no. 11YJCZH109), the Fundamental Research Funds for the Central Universities (nos. 30920130121006, 30920130132011), and the Zijin Intelligent Program in Nanjing University of Science and Technology (no. 2013-ZJ0211).

\section{References}

[1] N. P. Rachaniotis, T. K. Dasaklis, and C. P. Pappis, "A deterministic resource scheduling model in epidemic control: a case study," European Journal of Operational Research, vol. 216, no. 1, pp. 225-231, 2012.

[2] Z. Gao, D. Kong, and C. Gao, "Modeling and control of complex dynamic systems: applied mathematical aspects," Journal of Applied Mathematics, vol. 2012, Article ID 869792, 5 pages, 2012.

[3] B. K. Mishra and D. K. Saini, "SEIRS epidemic model with delay for transmission of malicious objects in computer network," 
Applied Mathematics and Computation, vol. 188, no. 2, pp. 14761482, 2007.

[4] C. J. Sun and Y.-H. Hsieh, "Global analysis of an SEIR model with varying population size and vaccination," Applied Mathematical Modelling, vol. 34, no. 10, pp. 2685-2697, 2010.

[5] M. Y. Li, J. R. Graef, L. C. Wang, and J. Karsai, "Global dynamics of a SEIR model with varying total population size," Mathematical Biosciences, vol. 160, no. 2, pp. 191-213, 1999.

[6] J. Zhang, J. Q. Li, and Z. Ma, "Global dynamics of an SEIR epidemic model with immigration of different compartments," Acta Mathematica Scientia B, vol. 26, no. 3, pp. 551-567, 2006.

[7] J. Zhang and Z. Ma, "Global dynamics of an SEIR epidemic model with saturating contact rate," Mathematical Biosciences, vol. 185, no. 1, pp. 15-32, 2003.

[8] K. I. Kim, Z. G. Lin, and L. Zhang, "Avian-human influenza epidemic model with diffusion," Nonlinear Analysis: Real World Applications, vol. 11, no. 1, pp. 313-322, 2010.

[9] J. L. Liu and T. L. Zhang, "Epidemic spreading of an SEIRS model in scale-free networks," Communications in Nonlinear Science and Numerical Simulation, vol. 16, no. 8, pp. 3375-3384, 2011.

[10] M. Samsuzzoha, M. Singh, and D. Lucy, "Numerical study of an influenza epidemic model with diffusion," Applied Mathematics and Computation, vol. 217, no. 7, pp. 3461-3479, 2010.

[11] M. Samsuzzoha, M. Singh, and D. Lucy, "A numerical study on an influenza epidemic model with vaccination and diffusion," Applied Mathematics and Computation, vol. 219, no. 1, pp. 122141, 2012.

[12] P. L. Shi and L. Z. Dong, "Dynamical models for infectious diseases with varying population size and vaccinations," Journal of Applied Mathematics, vol. 2012, Article ID 824192, 20 pages, 2012.

[13] H. W. Hethcote, "Qualitative analyses of communicable disease models," Mathematical Biosciences, vol. 28, no. 3-4, pp. 335-356, 1976.

[14] C. M. Kribs-Zaleta and J. X. Velasco-Hernández, "A simple vaccination model with multiple endemic states," Mathematical Biosciences, vol. 164, no. 2, pp. 183-201, 2000.

[15] L. S. Liebovitch and I. B. Schwartz, "Migration induced epidemics: dynamics of flux-based multipatch models," Physics Letters A, vol. 332, no. 3-4, pp. 256-267, 2004.

[16] A. Sani and D. P. Kroese, "Controlling the number of HIV infectives in a mobile population," Mathematical Biosciences, vol. 213, no. 2, pp. 103-112, 2008.

[17] Y. Yang, J. Wu, J. Li, and Z. Ma, "Global dynamics-convergence to equilibria-of epidemic patch models with immigration," Mathematical and Computer Modelling, vol. 51, no. 5-6, pp. 329337, 2010.

[18] M. Wolkewitz and M. Schumacher, "Simulating and analysing infectious disease data in a heterogeneous population with migration," Computer Methods and Programs in Biomedicine, vol. 104, no. 2, pp. 29-36, 2011.

[19] J. M. Lee, D. Choi, G. Cho, and Y. Kim, "The effect of public health interventions on the spread of influenza among cities," Journal of Theoretical Biology, vol. 293, pp. 131-142, 2012.

[20] M. Liu and Y. Xiao, "Modeling and analysis of epidemic diffusion within small-world network," Journal of Applied Mathematics, vol. 2012, Article ID 841531, 14 pages, 2012.

[21] M. Liu and L. Zhao, "Analysis for epidemic diffusion and emergency demand in an anti-bioterrorism system," International Journal of Mathematical Modelling and Numerical Optimisation, vol. 2, no. 1, pp. 51-68, 2011. 


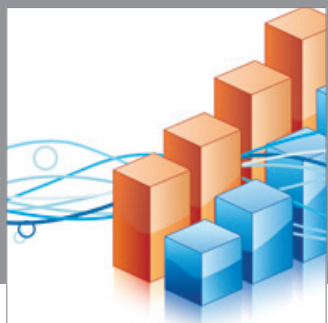

Advances in

Operations Research

mansans

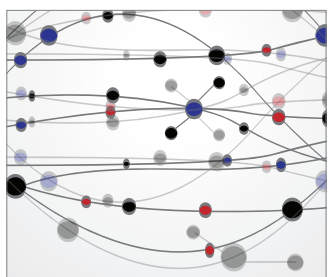

The Scientific World Journal
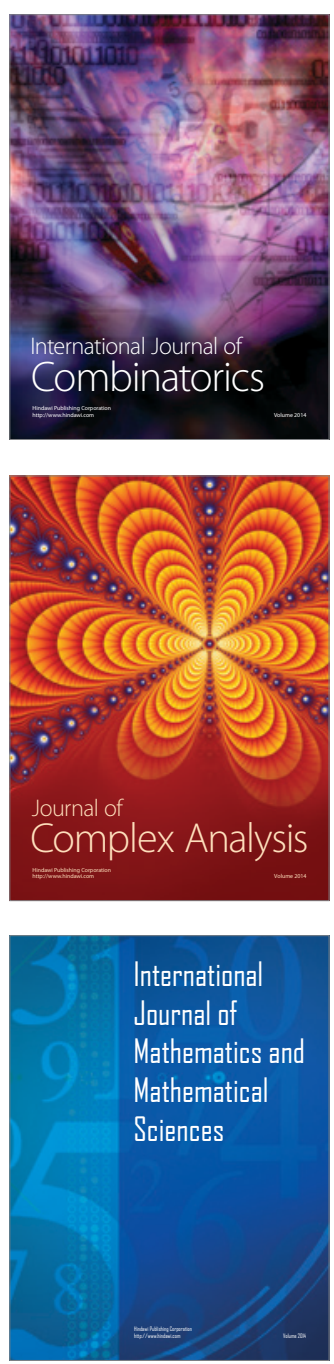


Submit your manuscripts at http://www.hindawi.com
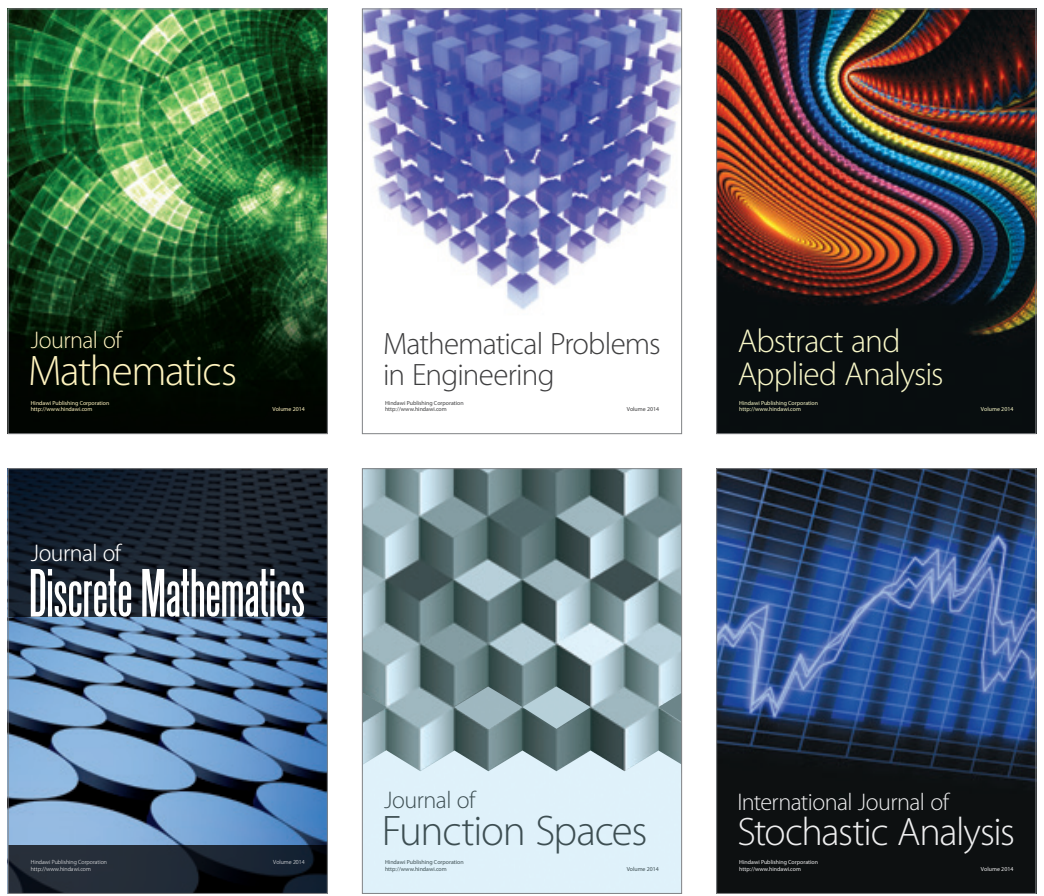

Journal of

Function Spaces

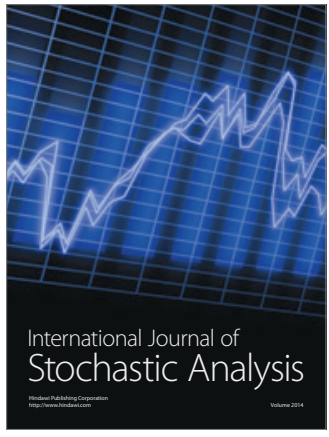

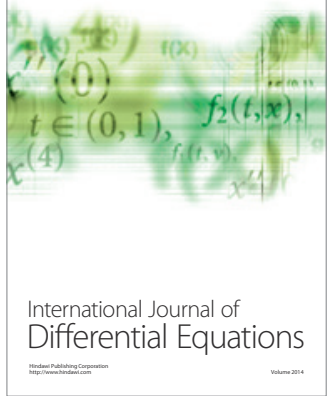
